\title{
NUMERICAL WORD-LENGTH OPTIMIZATION FOR CDMA DEMODULATOR
}

\author{
Kyungtae Han, Iksu Eo, Kyungsu Kim, Hanjin Cho \\ Electronics and Telecommunications Research Institute \\ 161 Kajong-dong, Yusong-Gu, Taejeon, 305-350, Korea \\ E-mail: $\{$ han, iseo, kimks, hjcho $\} @$ etri.re.kr
}

\begin{abstract}
This paper presents search methods to optimize word-length for digital systems. Finding the word-length is tedious work when the variables for optimization are numerous. We have proposed sequential and preplanned searches to find out optimum wordlength, and compared them in terms of the trials. A comparison for a given optimized point is evaluated. We apply them to word-length optimization for a CDMA demodulator, of which requirement is FER of 0.03. Our results show the sequential and the preplanned search have reduced the trials by the rate of $64 \%$ and $89 \%$, respectively compared to a full search to optimize the word-length for the CDMA demodulator design.
\end{abstract}

\section{INTRODUCTION}

Word-length is an important factor to implement digital signal processing systems. If the word-length is long enough, the performance is improved but hardware cost increases. If the word-length is short, the performance is degraded but the hardware cost decreases. There are many works for the wordlength optimization [1][2]. Since most systems cannot be optimized only by analytical methods, numerical search technique must be applied [3]. These methods are characterized by one essential idea namely to find a new feasible point, which is better than the existing one. These techniques are applied iteratively until no further improvement is possible or until the optimum has been located to within the desired accuracy.

Sung has numerically optimized the word-length for digital signal processing using exhaustive search [1]. However the exhaustive search for optimization is inefficient with regard to the trials for word-length optimization when the variables for optimization are numerous. We have proposed a sequential search and a preplanned search in order to reduce the trials. We have applied the results to optimize a code division multiple access (CDMA) demodulator that has numerous variables, and compared performance of each of the search methods

\section{WORD-LENGTH OPTIMIZATION}

\subsection{General Definition}

We define a word-length vector $\vec{w}$ that is composed of independent word-lengths as

$$
\vec{w}=\left(w_{1}, w_{2}, \ldots, w_{N}\right)
$$

where $N$ is the number of the variables to find out the optimum word-length in digital system [1]. The hardware cost-per-bit vector $\vec{c}$ has each of cost-per-bit components as

$$
\vec{c}=\left(c_{1}, c_{2}, \ldots, c_{N}\right) .
$$

The total cost is calculated by the dot-product of (1) with (2). The performance of digital systems, output signal to noise ratio (SNR), is represented by $p(\cdot)$. The optimum word-length vector $\vec{w}_{\text {opt }}$ is found when the hardware cost is minimized and the measured performance $p\left(\vec{w}_{\text {opt }}\right)$ is larger than the desired performance $p_{\text {desired }}$ as

$$
\begin{array}{ll}
\text { Minimize } & \vec{w}_{\text {opt }} \cdot \vec{c}^{T}, \\
\text { subject to } & p\left(\vec{w}_{\text {opt }}\right) \geq p_{\text {desired }}
\end{array}
$$

Optimization of the word-length has two procedures; first, finding base point of each variable in order to decide minimum word-lengths and then increasing the word-lengths from the base point according to direction of search.

\subsection{Base Point}

The base point in a variable is the lowest word-length satisfying the desired performance during one-variable simulation. Only one variable gradually is changed, while the word-length of other variables remains the same. The base point of the word-length is obtained by

$$
\begin{aligned}
& \vec{w}_{\text {base }}=\left(\min \left(w_{1}\right), \min \left(w_{2}\right), \ldots, \min \left(w_{N}\right)\right)
\end{aligned}
$$

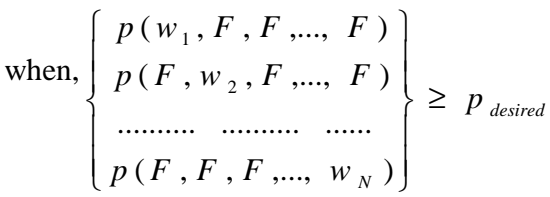

where $F$ is the full word-length, for example 16,32 or 64 bits.

\subsection{Direction of Search}

\subsubsection{Full Search}

Sung and Kum used the full search algorithm exhaustively searching feasible locations for the low hardware cost from the base point until the optimum has been located to within the desired accuracy 1 .

For example, we assume the numbers of the independent variables to find optimum word-length $N$ are two, and the base 
point is $(2,2)$, and each of word-length cost is similar. In the full search, the direction of search is shown in Fig. 1. An optimized point $(5,5)$ is given for a comparison between three methods. The minimum number of experiments is 24 by count.

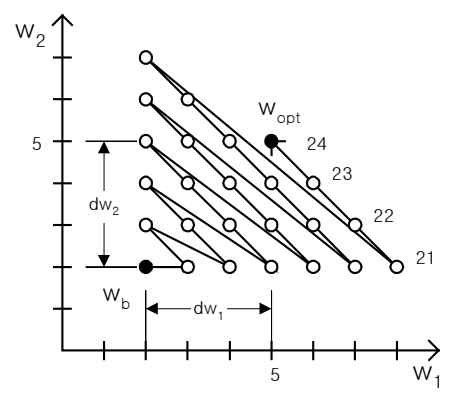

Figure 1. The direction of full search (base point $=2,2$; optimum point $=5,5 ; \mathrm{d}=6$; trials $=24$ )

We have generalized the total number of experiments of the full search in $\mathrm{N}$-dimension using summation of Combination-withReplacement as [4]

$$
\begin{aligned}
E_{F S}^{N}(d) & =\sum_{r=0}^{d-1} \mathrm{C}^{\mathrm{R}}(N, r) \\
& =\mathrm{C}^{\mathrm{R}}(N+1, d-1) \\
& =\left(\begin{array}{c}
N+d-1 \\
d-1
\end{array}\right) \\
& =\frac{(d+N-1) \ldots(d+2)(d+1) d}{N !} \\
\text { where } \quad d & =d w_{1}+d w_{2}+\ldots+d w_{N} .
\end{aligned}
$$

The range of the trials of the full search with distance $d$ in $\mathrm{N}$ dimension are

$$
E_{F S}^{N}(d) \leq E_{F S}^{N, d}<E_{F S}^{N}(d+1) .
$$

\subsubsection{Sequential Search (SS)}

The basic notion of sequential search is that each trial eliminates a portion of the region being searched. The sequential search method decides where the most promising areas are located, and continues in the most favorable region after each set of experiments [3].

The principles of sequential search in $n$ dimensions can be summarized in the following four steps:

1. Select a set of feasible values for the independent variables, which satisfy the desired performance during one-variable simulation. This is a base point.

2. Evaluate the performance at the base point.

3. Choose feasible locations at which evaluate the performances and compare their performance.

4. If one point is better than others, move to the better point, and repeat the search, until the point has been located to within the desired accuracy.

In step 3, the direction of search is chosen in accordance with maximum derivative of their performance:

$$
\vec{w}_{j+1}=\vec{w}_{j}+s \vec{\xi}_{j}
$$

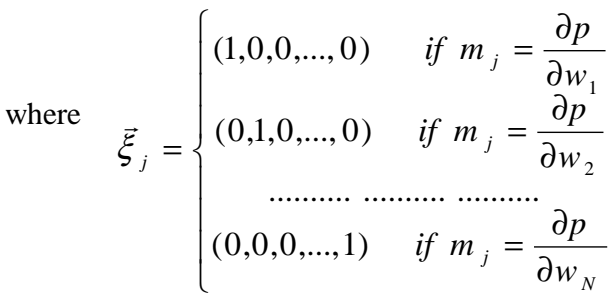

$$
\begin{aligned}
& \text { and } m_{j}=\max \left(\frac{\partial p}{\partial w_{1}}, \frac{\partial p}{\partial w_{2}}, \ldots ., \frac{\partial p}{\partial w_{N}}\right)
\end{aligned}
$$

and $S$ is step size.

We use the same condition of the full search for comparison. In Fig. 2, starting from base point $(2,2)$, we measure performance of $(2,3)$ and $(3,2)$ from the direction of sequential search in step 3. If the performance of $(3,2)$ is better than that of $(2,3)$, move the point into $(3,2)$ that is a new word-length set. Simulation would be repeated until satisfying the desired performance.

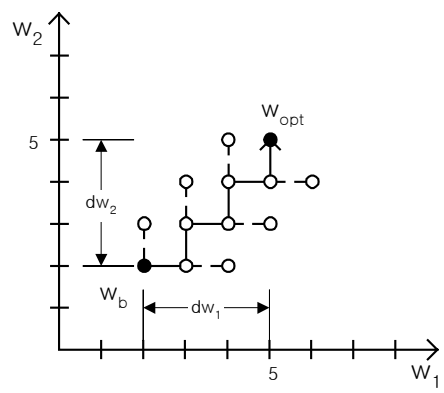

Figure 2. The direction of sequential search (base point $=2,2$; optimum point $=5,5$; trials $=12$ )

We have generalized the trials of the sequential search in $\mathrm{N}$ dimension as

$$
E_{S S}^{N}=N \cdot\left(d w_{1}+d w_{2}+\ldots+d w_{N}\right) .
$$

In this example, the trials are 12 from (12) and also 12 from Fig. 2 by count.

\subsubsection{Preplanned Search (PS)}

A preplanned search is one in which all the experiments are completely scheduled in advance, and the results do not and cannot influence the course of these experiments. The preplanned directions are obtained from the sensitivity of performance of an independent variable before experiments. The greatest rate of improvement is found out by moving along the greatest gradient among them.

The principles of preplanned search in $n$ dimensions are summarized in the following four steps:

1. Select a set of feasible values for the independent variables, which satisfy the desired performance during one-variable simulation. This is a base point.

2. Preplan, by a method to be described, feasible locations.

3. Evaluate the performance at those points.

4. Move to the preplanned points, until the point has been located to within the desired accuracy. 
In step 2, the direction of preplanned search is chosen in accordance with maximum derivative of an independent performance:

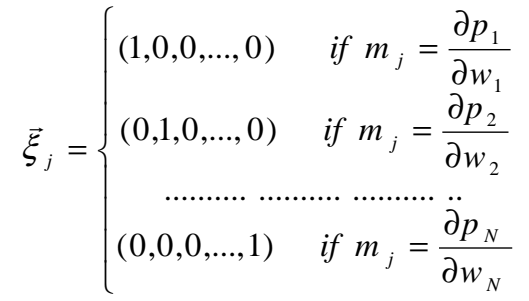

$$
\begin{aligned}
& \text { where } m_{j}=\max \left(\frac{\partial p_{1}}{\partial w_{1}}, \frac{\partial p_{2}}{\partial w_{2}}, \ldots ., \frac{\partial p_{N}}{\partial w_{N}}\right)
\end{aligned}
$$

We use the same condition of the full search for comparison. In Fig. 3, starting from base point $(2,2)$, we preplan the directions of the steepest ascent by comparing the gradients of the independent performances at one dimension from one-variable simulation. If the gradient, calculated from the one-variable simulation in section 2.2, at $w_{1}$ of 2 is larger than that at $w_{2}$ of 2 , the next feasible location is $(3,2)$. Then, if the gradient at $w_{1}$ of 3 is smaller than that at $w_{2}$ of 2 , the next feasible location is $(3,3)$. The preplanned path would be $(2,2),(3,2),(3,3)$, etc. After scheduling the feasible points, evaluate the performance of these points, until the value of the performance meets the desired accuracy.

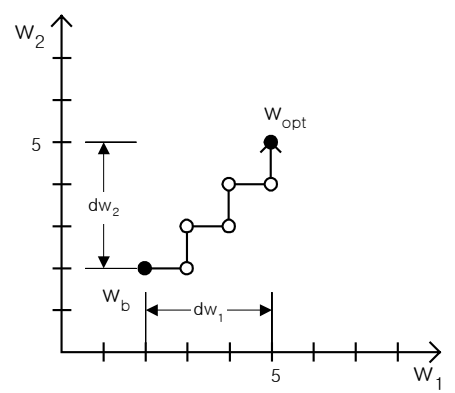

Figure 3. The direction of preplanned search (base point $=2,2$; optimum point $=5,5$; trials $=6$ )

We generalized the trials of the preplanned search in $\mathrm{N}$ dimension as

$$
E_{P S}^{N}=d w_{1}+d w_{2}+\ldots+d w_{N}
$$

In this example, the trials are 6 from (15) and 6 from Fig. 3 by count.

\section{SIMULATION}

We applied the proposed optimization methods to CDMA demodulator design.

\subsection{CDMA Demodulator}

Typical demodulators are implemented with an analog block in front of an analog digital converter (ADC) block as shown in fig.4 (a). As the speed of the ADC increases, analog parts are replaced with digital parts in communication systems [5]. We replaced the analog demodulator with the digital demodulator as shown in Fig. 4 (b).
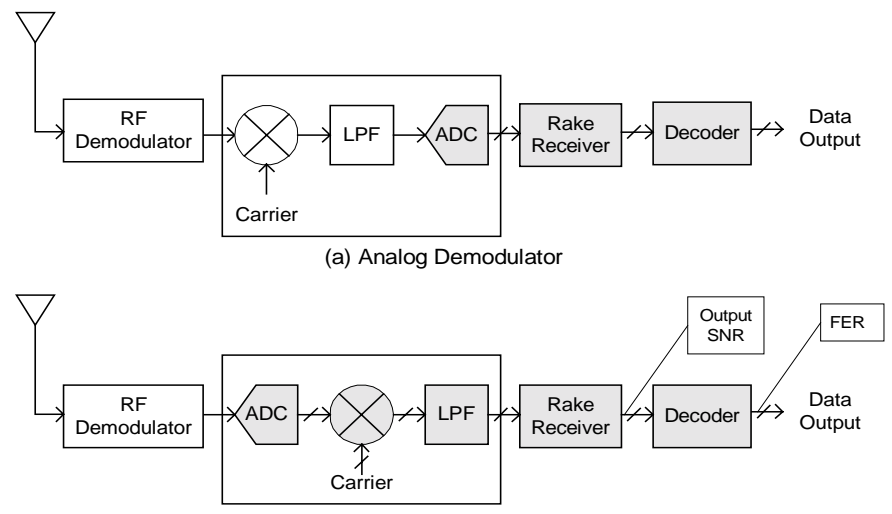

(b) Digital Demodulator

Figure 4. Analog and digital demodulator in CDMA receiver and performance measurement position

The demodulator converts modulated signals into baseband signals. In the digital demodulator block of Fig. 5, the sampled data at the ADC are multiplied by a carrier signal down to the baseband signal. The out-of-band signal is removed

by the low pass filter (LPF). The variables in the digital demodulator are as below [6]:

- $B_{i}$ : input word-length

- $B_{c}$ : carrier word-length

- $B_{m}:$ multiplier output word-length

- $B_{f}$ : filter output word-length

- $B_{f c}:$ filter coefficient word-length.

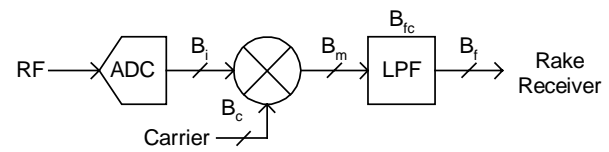

Figure 5. A digital demodulator block.

We have used the output SNR instead of frame error rate (FER), which is a general measurement to evaluate CDMA systems because direct measurement of FER requires at least $10^{5}$ data during simulation [7]. The required output SNR in this system is over $0.8 \mathrm{~dB}$ or FER is under 0.03 [8].

Commonly the base point of $\left(B_{i}, B_{c}, B_{m}, B_{f}, B_{f c}\right)$ over $0.8 \mathrm{~dB}$ is $(4,3,4,5,7)$, acquired from the one-variable simulations shown as Fig 6. We assume cost-per-bit is similar. In the FS, the next points are exhaustively $(5,3,4,5,7),(4,4,4,5,7),(4,3,5,5,7)$, $(4,3,4,6,7),(4,3,4,5,8),(5,4,4,5,7)$ and etc. The search is continued until the performance meets the specific desired requirement.

In the SS, the next point is one of $(5,3,4,5,7),(4,4,4,5,7)$, $(4,3,5,5,7),(4,3,4,6,7)$ and $(4,3,4,5,8)$. The next point would be the set having the maximum performance of them. From table 1, $(4,3,4,6,7)$ is the next location because it has the maximum performance. Move into the new point and continue to search until the performance is over the specific desired requirement, output SNR of 0.8 . The optimum point is $(5,3,6,6,7)$. The distance between the base and the optimum point is 4 . 


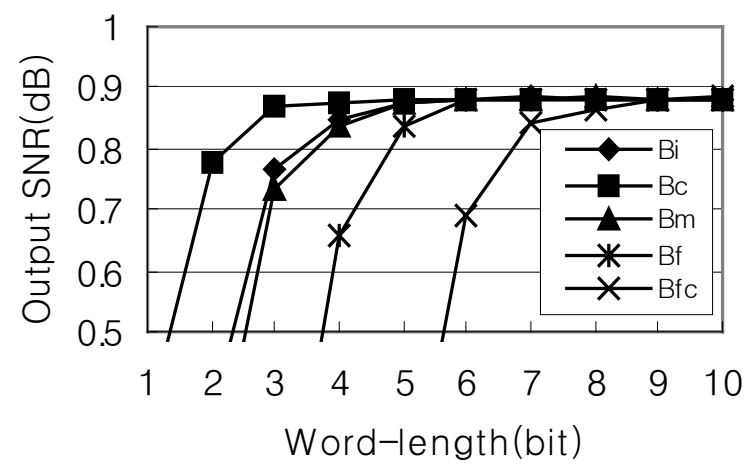

Figure 6. Result of independent one-variable simulations on a CDMA demodulator

In the PS, search path is estimated from the sensitivity of each one-variable simulation shown in Fig. 6. Starting from the base point $(4,3,4,5,7)$, the first expected point is $(4,3,4,6,7)$ because the $B_{f}$ has the greatest derivative among each word-length at the base point from Fig. 6. The preplanned search points are $(4,3,4,5,7),(4,3,4,6,7),(4,3,4,6,8),(4,3,5,6,8),(4,4,5,6,8)$, etc. Move into the preplanned points until the performance is over the specific desired requirement. The optimum point is $(5,4,5,6,8)$ and distance is 6 .

\subsection{COMPARISON}

The three search methods are compared with the trials from (6), (12), (15), as shown in table 2. The FS is inefficient to find the optimum word-length when the variables for optimization are numerous and the distance between base and optimum point is long. The SS is easy to be applied to digital systems and has fewer numbers of experiments. The PS, which needs the calculation of the gradients of the performance at each variable, has the least number of experiments among search methods. But, the SS and PS have a loss of direction problem encountered by techniques based on the gradient projection method. This problem can be solved by an adaptive step size. The SS and the PS reduced the trials by the rate of $64 \%$ and $89 \%$, respectively, compared to the FS in word-length optimization for the CDMA demodulator design. However, PS seldom converges to the same optimum point and the distance is longer than others.

\section{CONCLUSION}

In this paper, numerical search methods to optimize the wordlength for digital implementations are explained. We consider the FS and propose SS and FS. We have generalized the trials of them as (6), (12), (15). The FS is inefficient with regard to the trials. The SS requires fewer experiments and the PS needs the least experiments when the variables for optimization are numerous. We have applied those methods to a CDMA demodulator design. The simulation results show the SS and PS reduced the number of trials by the rate of $64 \%, 89 \%$ respectively compared to full search finding the optimum wordlength in the CDMA demodulator design. However, PS has longer distance than others. In the future, an adaptive step size for the loss of direction and fast search would be considered.
Table 1. Sequence of the Sequential Search for CDMA demodulator (Traffic Ch. Rate Set 1 in AWGN, Input SNR $=-17.3 \mathrm{~dB}, \mathrm{~Eb} / \mathrm{Nt}=3.8$, Rate $=9600$ bps, Desired performance: Output $\mathrm{SNR}>0.8$, FER $<0.03$ )

\begin{tabular}{|c|c|c|c|c|c|c|c|c|}
\hline Step & $\mathrm{Bi}$ & $\mathrm{Bc}$ & $\mathrm{Bm}$ & $\mathrm{Bf}$ & $\mathrm{Bfc}$ & Output SNR & FER & Result \\
\hline 1,2 & 4 & 3 & 4 & 5 & 7 & 0.711 & 0.0384 & Fail \\
\hline 3 & 5 & 3 & 4 & 5 & 7 & 0.735 & - & - \\
\hline 3 & 4 & 4 & 4 & 5 & 7 & 0.694 & - & - \\
\hline 3 & 4 & 3 & 5 & 5 & 7 & 0.712 & - & - \\
\hline 3 & 4 & 3 & 4 & 6 & 7 & 0.759 & - & $\operatorname{Max}$ \\
\hline 3 & 4 & 3 & 4 & 5 & 8 & 0.704 & - & - \\
\hline 4 & 4 & 3 & 4 & 6 & 7 & 0.759 & 0.035 & Fail \\
\hline 3 & 5 & 3 & 4 & 6 & 7 & 0.763 & - & - \\
\hline 3 & 4 & 4 & 4 & 6 & 7 & 0.722 & - & - \\
\hline 3 & 4 & 3 & 5 & 6 & 7 & 0.773 & - & $\operatorname{Max}$ \\
\hline 3 & 4 & 3 & 4 & 7 & 7 & 0.751 & - & - \\
\hline 3 & 4 & 3 & 4 & 6 & 8 & 0.749 & - & - \\
\hline 4 & 4 & 3 & 5 & 6 & 7 & 0.773 & 0.0342 & Fail \\
\hline \multicolumn{9}{|c|}{ 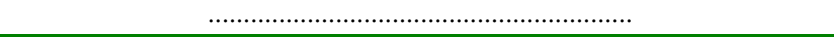 } \\
\hline 3 & 6 & 3 & 5 & 6 & 7 & 0.798 & - & - \\
\hline 3 & 5 & 4 & 5 & 6 & 7 & 0.802 & - & - \\
\hline 3 & 5 & 3 & 6 & 6 & 7 & 0.805 & - & Max \\
\hline 3 & 5 & 3 & 5 & 7 & 7 & 0.803 & - & - \\
\hline 3 & 5 & 3 & 5 & 6 & 8 & 0.798 & - & - \\
\hline 4 & 5 & 3 & 6 & 6 & 7 & 0.805 & 0.0292 & Pass \\
\hline \multicolumn{9}{|c|}{ Table 2. Comparison of full, sequential and preplanned searches $(\mathrm{N}=5$, ) } \\
\hline Type & \multicolumn{2}{|c|}{$\begin{array}{l}\text { Distance } \\
(\mathrm{d})\end{array}$} & \multicolumn{5}{|c|}{$\begin{array}{c}\text { Equation for Number of Experiments } \\
\text { from }(6),(12),(15)\end{array}$} & Trials \\
\hline FS & \multicolumn{2}{|c|}{ 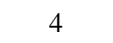 } & \multicolumn{5}{|c|}{$(d+4)(d+3)(d+2)(d+1) \cdot d / 5 !$} & 56 \\
\hline SS & \multicolumn{2}{|c|}{4} & \multicolumn{5}{|c|}{$5 \cdot d$} & 20 \\
\hline PS & \multicolumn{2}{|c|}{6} & \multicolumn{5}{|c|}{$d$} & 6 \\
\hline
\end{tabular}

\section{References}

[1] W. Sung and K. Kum, "Simulation-based word-length optimization method for fixed-point digital signal processing systems," IEEE Trans. on Signal Processing, vol. 43, pp. 3087-3090, Dec. 1995.

[2] S. A. Wadekar and A. C.Parker, "Accuracy Sensitive Word-length Selection for Algorithm Optimization," Int'l Conf. on Computer Design, pp. 54-61, 1998.

[3] G.S.G. Beveridge, and R.S. Schechter, Optimization: Theory and Practice, McGraw-Hill, Tokyo, 1970.

[4] K.H. Rosen, Handbook of Discrete and Combinatorial Mathematics, CRC Press, 2000.

[5] J.A. Wepman, "Analog-to-digital converters and their applications in radio receivers," IEEE Commum. Mag.,vol. 33, pp. 39-45, May 1995.

[6] S. Kim, K. Han and W. Sung, "A CORDIC-based Digital Quadrature Mixer: Comparison with ROM-based Architecture," IEEE Int'l Symp. on Circuits and Systems, vol. 4, pp. 385 - 388, Monterey, CA, Jun., 1998.

[7] J-S. Wu, M-L. Liou, H-P. Ma and T-D. Chiueh, "A 2.6V, 44-MHz All-Digital QPSK Direct-Sequence SpreadSpectrum Transceiver IC," IEEE J. of Solid-State Circuits, vol. 32, no.10, pp. 1499-1510, Oct., 1997.

[8] K. Han, I. Eo, K. Kim and H. Cho, "Bit Constraint Parameter Decision Method for CDMA Digital Demodulator," The $5^{\text {th }}$ CDMA Int'l. Conf. \& Exhibit., vol. 2, pp. 583-586, Seoul, Nov., 2000. 